\title{
Video-rate photoacoustic microscopy of micro-vasculatures
}

Lidai Wang, Konstantin Maslov, Wenxin Xing, Alejandro Garcia-Uribe, Lihong V. Wang

Lidai Wang, Konstantin Maslov, Wenxin Xing, Alejandro Garcia-Uribe, Lihong V. Wang, "Video-rate photoacoustic microscopy of micro-vasculatures ," Proc. SPIE 8581, Photons Plus Ultrasound: Imaging and Sensing 2013, 85811T (4 March 2013); doi: 10.1117/12.2004714

SPIE. Event: SPIE BiOS, 2013, San Francisco, California, United States 


\title{
Video-rate photoacoustic microscopy of micro-vasculatures
}

\author{
Lidai Wang, Konstantin Maslov, Wenxin Xing, Alejandro Garcia-Uribe and Lihong V. Wang* \\ Optical Imaging Laboratory, Department of Biomedical Engineering, Washington University in St. \\ Louis. Campus Box 1097, One Brookings Drive, St. Louis, Missouri 63130-4899, USA
}

\begin{abstract}
We report the development of photoacoustic microscopy capable of video-rate high-resolution invivo imaging in deep tissue. A lightweight photoacoustic probe is made of a single-element broadband ultrasound transducer, a compact photoacoustic beam combiner, and a bright-field light delivery system. Focused broadband ultrasound detection provides a $44-\mu \mathrm{m}$ lateral resolution and a 28- $\mu \mathrm{m}$ axial resolution. A multimode optical fiber is used to deliver laser pulses. The bright-field light delivery system can effectively improve the illumination efficiency. The photoacoustic probe weighs less than 40 grams and is mounted on a voice-coil scanner to acquire 40 cross-sectional images per second over several-mm range. The fast speed can effectively improve imaging throughput, reduce motion artifacts, and enable the visualization of highly dynamic biomedical processes. High-resolution micro-vascular imaging is successfully demonstrated.
\end{abstract}

Keywords: Photoacoustic imaging, fast scanning speed, deep imaging, cardiovascular imaging

\section{INTRODUCTION}

Photoacoustic (PA) imaging offers high spatial resolution and unique optical absorption contrasts in deep tissue (1). Different PA imaging systems have demonstrated anatomical, functional, and molecular imaging up to several millimeters and even centimeters deep in vivo (2-10). A high imaging speed is important in order to apply this emerging technique to broader clinical practice and biomedical studies. Fast PA imaging can bring many benefits, such as enabling the study of dynamic physiological or pathological processes, reducing motion artifacts, and shortening imaging time.

A major modality of PA imaging, acoustic-resolution photoacoustic microscopy (AR-PAM), mechanically scans a single focused ultrasound transducer to form two-dimensional and threedimensional images. Recently, the imaging speed of AR-PAM was increased to $10 \mathrm{~Hz}$ over a 9-mm scanning range using a voice-coil stage (11). However, due to a low laser repetition rate, only slow PA imaging was implemented. For deep, fast AR-PAM imaging, a high pulse-repetition-rate laser with enough pulse energy is required. However, it is technically challenging and cost-prohibitive to manufacture a high pulse energy laser with tens of $\mathrm{kHz}$ pulse repetition rate. Therefore, an efficient light-delivery system is necessary for fast AR-PAM.

Photons Plus Ultrasound: Imaging and Sensing 2013, edited by Alexander A. Oraevsky, Lihong V. Wang, Proc. of SPIE Vol. 8581, 85811T - C 2013 SPIE · CCC code: 1605-7422/13/\$18 - doi: 10.1117/12.2004714 
Here, we developed a fast AR-PAM system based on a lightweight PA probe and a voice-coil scanner. The PA probe is comprised mainly of a high-frequency broadband ultrasound transducer, a custom-made PA beam combiner, and a bright-field light delivery system. The light delivery system focuses the light beam tighter on the tissue surface, so that there is a higher photon delivery efficiency. The optical and acoustic axes are confocally and coaxially aligned through the PA beam combiner to achieve high detection efficiency. This design improves light delivery efficiency and lowers the required pulse energy more than ten times. Video-rate $(>30 \mathrm{~Hz})$ cross-sectional imaging is achieved over a 9-mm scanning range. Using a fast pulsed laser, the mouse micro-vasculature is imaged to demonstrate the real-time imaging capability.

\section{IMAGING SYSTEM DESIGN}

A fast Q-switch laser (wavelength: $532 \mathrm{~nm}$, pulse width: $10 \mathrm{ns,} 30 \mathrm{kHz}$, EdgeWave GmbH, Wurselen, Germany) delivers light to the PA probe through a multimode optical fiber. Maximum pulse energies of $30 \mu \mathrm{J}$ are employed for in vivo imaging. As shown in Figure 1, the light beam diverges from the output of the multimode fiber and then is weakly focused by an optical lens. A custom-made PA beam combiner, made of two right-angle glass prisms with a thin layer of aluminum coating in the middle, redirects the light beam onto the imaged objects. Because aluminum coating has an acoustic impedance similar to that of glass, the PA beam combiner effectively transmits the acoustic beam and reflects the optical beam. A plano-concave ultrasound lens is attached to the PA beam combiner to convert spherical ultrasound waves to planar waves. After passing through the PA beam combiner, the acoustic wave is received by a high-frequency broadband ultrasound transducer. The PA signal from the ultrasound transducer is amplified and then digitized at a 500-MHz sampling rate. The entire PA probe, as shown in Figure 1, weighs less than 40 grams, which makes it easy to be translated in high speed without causing strong vibrations.

A voice-coil stage is employed to scan the PA probe in the fast scanning axis (x-axis). A stepper motor stage translates the entire voice-coil stage in the slow scanning axis (y-axis). After each laser pulse, the system records a depth-resolved single (A-line) according to the acoustic propagation speed in soft tissue $(1.54 \mu \mathrm{m} / \mathrm{ns})$. Scanning the PA

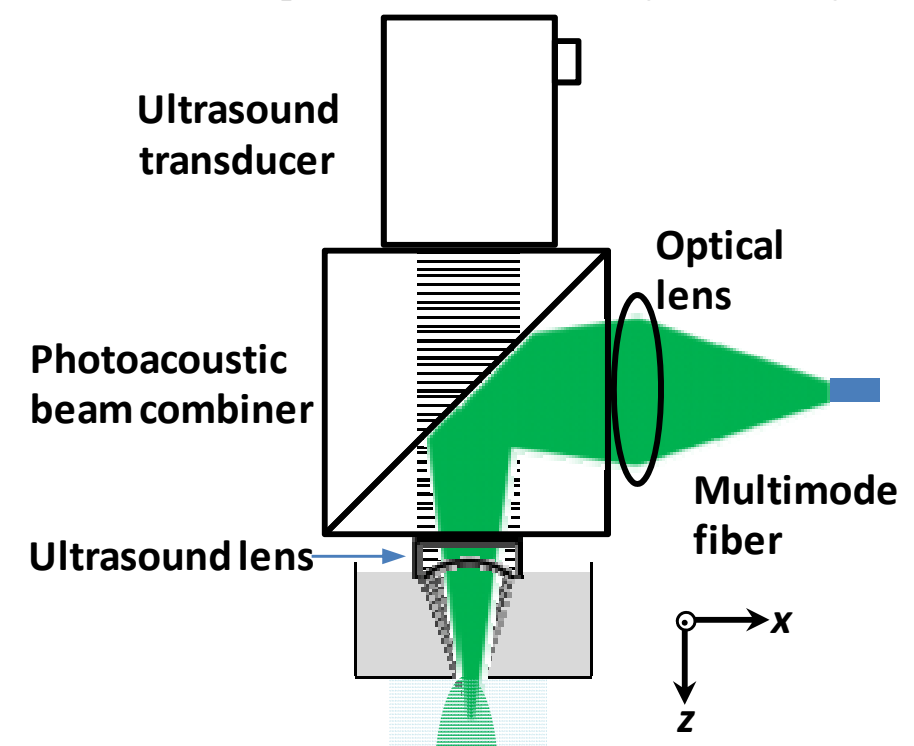

Figure 1. schematics of the fast AR-PAM system. 
probe along the x-axis generates cross-sectional (B-scan) images. Due to the light weight of the PA probe and the high force-to-mass ratio of the voice-coil actuator, the imaging system can acquire 40 B-scan images per second over a scanning range of $9 \mathrm{~mm}$. A field-programmable-gate-array (FPGA) card is used to generate synchronized laser triggers, digitizer triggers, and motion control commands.

\section{BRIGHT FIELD ILLUMINATION}

Monte Carlo simulation is used to study the AR-PAM system's illumination efficiency in deep tissue. The illuminated spot on the tissue surface had a diameter of $2 \mathrm{~mm}$. The incident pulse energy was set to unity. The tissue optical properties were set as refractive index $\mathrm{n}=1.33$, absorption coefficient $\mu_{\mathrm{a}}=0.1 \mathrm{~cm}^{-1}$, scattering coefficient $\mu_{\mathrm{s}}=100 \mathrm{~cm}^{-1}$, and scattering anisotropy $\mathrm{g}=0.9$. Figure 2 shows the relative fluence distributions of the bright-illuminations. The results indicate that, at several millimeters deep, photons can be effectively confined near the center of the illumination spot. Since pulse energy is usually limited in high-repetition-rate pulsed lasers, the weakly-focused bright-field illumination can improve the photon delivery efficiency.

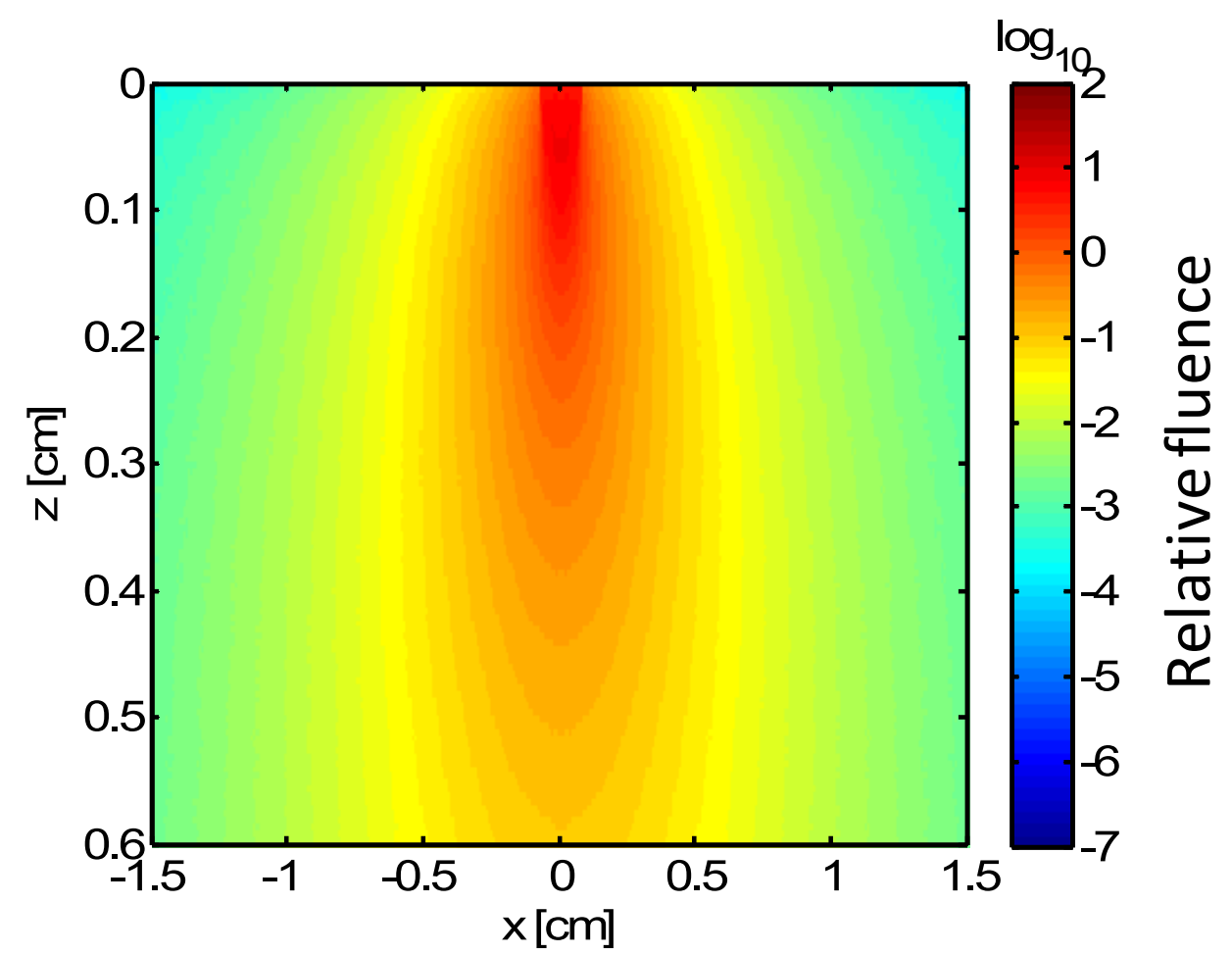

Figure 2. Monte Carlo simulation of the bright field illumination

\section{SYSTEM CHARACTERIZATION AND IN VIVO IMAGING}

The spatial resolution of the imaging system was determined by the acoustic focus. We measured it by imaging a carbon fiber with a $6-\mu \mathrm{m}$ diameter in water. The carbon fiber was placed parallel to the 
$y$-axis. The cross-sectional profile had a full width at half maximum (FWHM) of $44 \mu \mathrm{m}$. The axial (z-axis) resolution was determined by the ultrasound detection bandwidth. We quantified it by taking the FWHM of the envelope of an averaged A-line signal. Experimental result from the A-line signals of the carbon fiber provides an axial resolution better than $28 \mu \mathrm{m}$.

Micro-vasculatures of a mouse ear were imaged in vivo at 532-nm wavelength. In the in vivo experiments, nude mice were imaged under anesthesia. The mice were placed on a $37^{\circ} \mathrm{C}$ heating pad and secured with a nose cone. Ultrasound gel was applied between the tissue surface and the membrane, attached to an opening at the bottom of the water tank, to transmit ultrasound waves. All experimental animal procedures were carried out in conformance with the laboratory animal protocol approved by the Animal Studies Committee of Washington University in St. Louis. As shown in Figure 3, artery-vein pairs can be clearly identified.

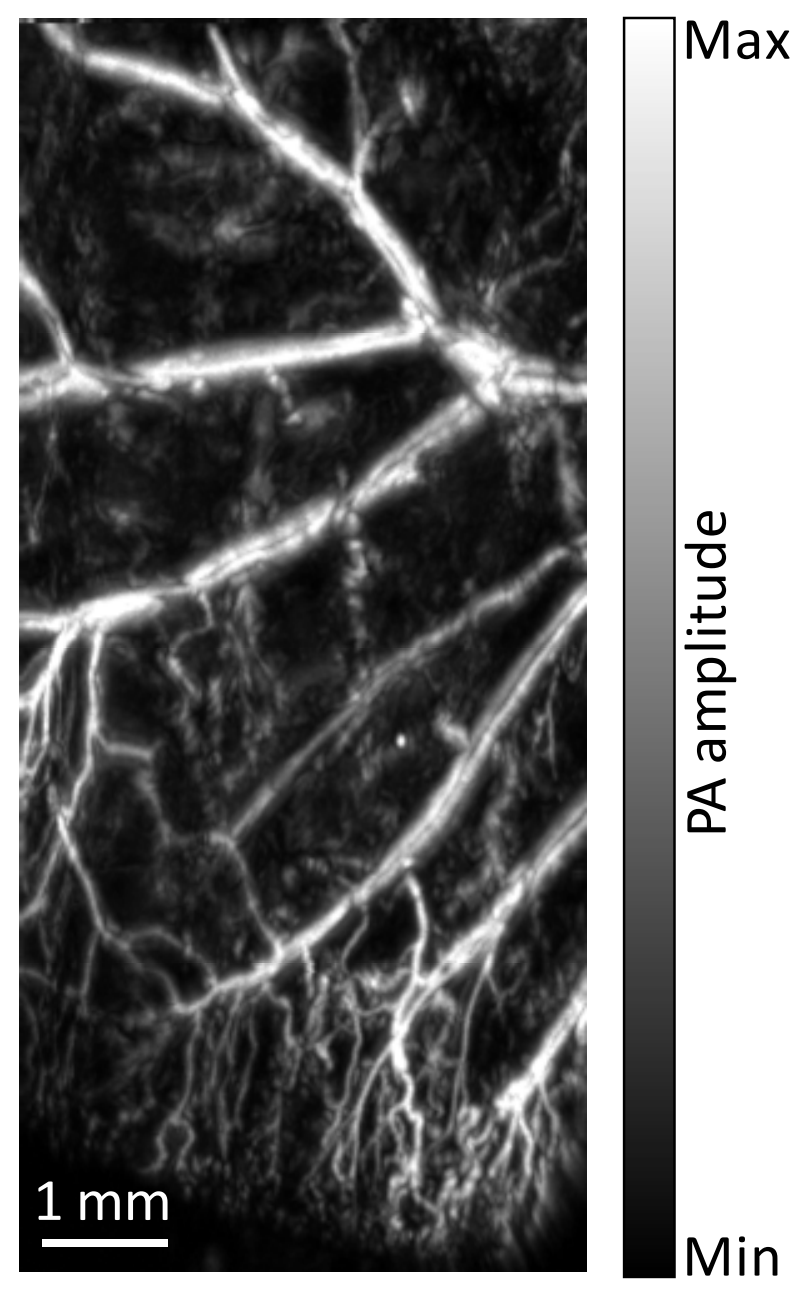

Figure 3. In vivo imaging of mouse ear micro-vasculature 


\section{VIDEO-RATE IMAGING OF MOUSE CARDIO-VASCULATURE}

The mouse was set in a supine position so that its chest wall faced up towards the PA probe. The laser was operated at a $532-\mathrm{nm}$ wavelength with a $28-\mu \mathrm{J}$ pulse energy. B-scan images were acquired at 40 frames per second. From the video-rate images, we can clear see the breathing motion. The breathing was measured from the displacement of the top vascularture surface. The breathing period and surface motion amplitude were quantified. Each measurement was obtained from one B-scan image. Figure 4 shows the inhalation gas was alternated between $5 \% \mathrm{O}_{2}$ (denoted as $\mathrm{A}$ in figure 4) and $100 \% \mathrm{O}_{2}$ (denoted as B in figure 4). During the transition from low $\mathrm{O}_{2}$ to high $\mathrm{O}_{2}$ inhalation gas, the breathing period clearly increased, and the breathing motion amplitude decreased. During the transition from high $\mathrm{O}_{2}$ to low $\mathrm{O}_{2}$, the breathing period became shorter, and the breathing motion became stronger.

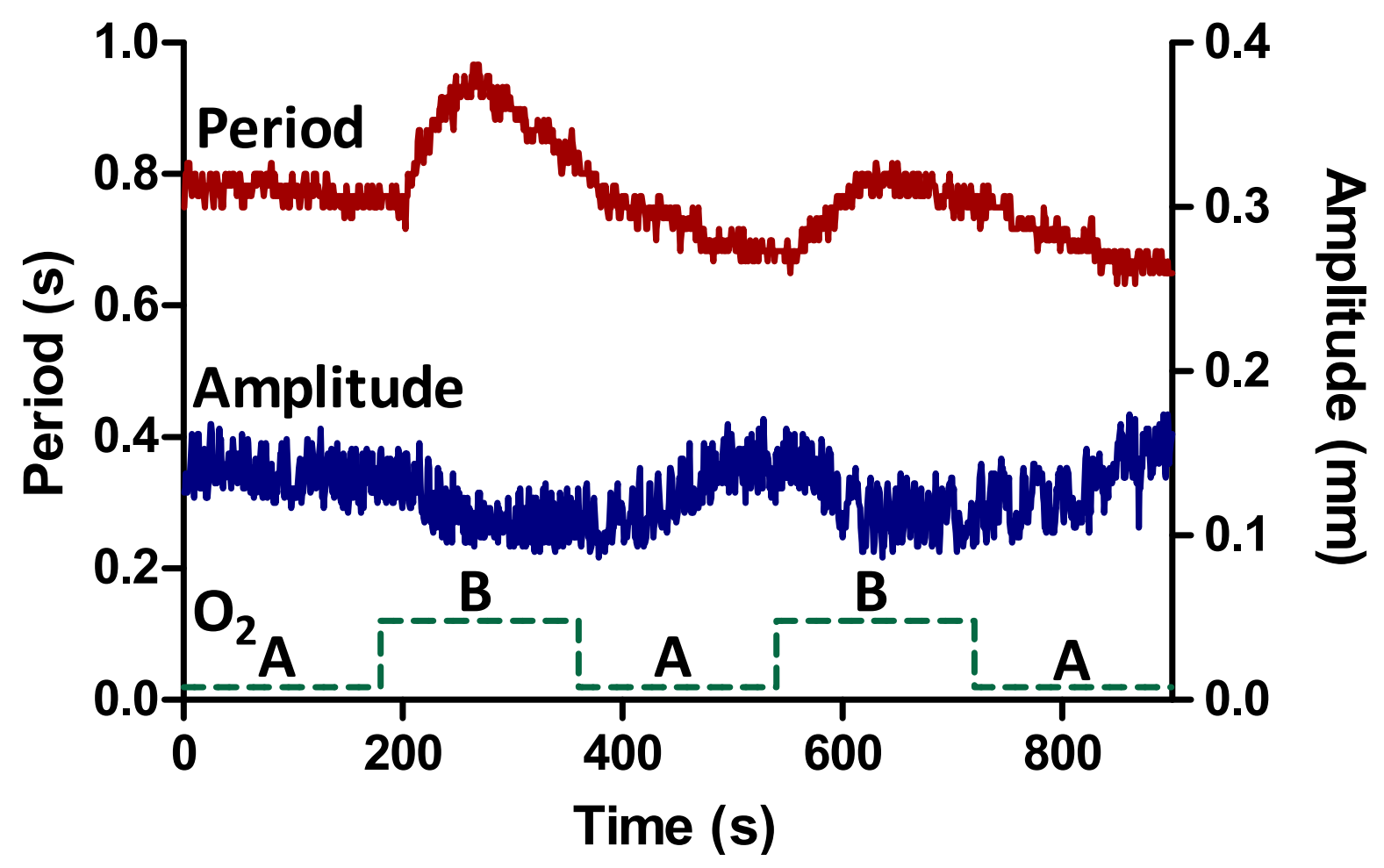

Figure 4. Breathing motion changes with inhalation gas

\section{CONCLUSION}

In summary, we have successfully developed a fast AR-PAM system which enabled video-rate imaging. A lightweight PA probe and a voice-coil scanner were employed for fast mechanical scanning. Bright-field illumination provided high photon delivery efficiency, which enabled the use of high-repetition-rate low-energy pulsed lasers to implement fast AR-PAM imaging. Imaging of the mouse ear and chest micro-vasculatures was demonstrated in vivo. 


\section{REFERENCES}

[1] L. V. Wang and S. Hu, "Photoacoustic Tomography: In Vivo Imaging from Organelles to Organs," Science 335(6075), 1458-1462 (2012)

[2] X. Wang, Y. Pang, G. Ku, X. Xie, G. Stoica and L. V. Wang, "Noninvasive laser-induced photoacoustic tomography for structural and functional in vivo imaging of the brain," Nat Biotech 21(7), 803$806(2003)$

[3] H. F. Zhang, K. Maslov, G. Stoica and L. V. Wang, "Functional photoacoustic microscopy for high-resolution and noninvasive in vivo imaging," Nat Biotech 24(7), 848-851 (2006)

[4] K. H. Song and L. V. Wang, "Deep reflection-mode photoacoustic imaging of biological tissue," Journal of Biomedical Optics 12(6), 060503-060503 (2007)

[5] L. Meng-Lin, O. Jung-Taek, X. Xueyi, K. Geng, W. Wei, L. Chun, G. Lungu, G. Stoica and L. V. Wang, "Simultaneous Molecular and Hypoxia Imaging of Brain Tumors In Vivo Using Spectroscopic Photoacoustic Tomography," Proceedings of the IEEE 96(3), 481-489 (2008)

[6] A. De La Zerda, C. Zavaleta, S. Keren, S. Vaithilingam, S. Bodapati, Z. Liu, J. Levi, B. R. Smith, T.-J. Ma, O. Oralkan, Z. Cheng, X. Chen, H. Dai, B. T. Khuri-Yakub and S. S. Gambhir, "Carbon nanotubes as photoacoustic molecular imaging agents in living mice," Nat Nano 3(9), 557-562 (2008)

[7] C. Kim, T. N. Erpelding, L. Jankovic, M. D. Pashley and L. V. Wang, "Deeply penetrating in vivo photoacoustic imaging using a clinical ultrasound array system," Biomed. Opt. Express 1(1), 278-284 (2010)

[8] G. S. Filonov, A. Krumholz, J. Xia, J. Yao, L. V. Wang and V. V. Verkhusha, "Deep-Tissue Photoacoustic Tomography of a Genetically Encoded Near-Infrared Fluorescent Probe," Angewandte Chemie International Edition 51(6), 1448-1451 (2012)

[9] L. Wang, K. Maslov, J. Yao, B. Rao and L. V. Wang, "Fast voice-coil scanning optical-resolution photoacoustic microscopy," Opt. Lett. 36(2), 139-141 (2011)

[10] K. J. Rowland, J. Yao, L. Wang, C. R. Erwin, K. I. Maslov, L. V. Wang and B. W. Warner, "Immediate alterations in intestinal oxygen saturation and blood flow after massive small bowel resection as measured by photoacoustic microscopy," Journal of Pediatric Surgery 47(6), 1143-1149 (2012)

[11] T. Harrison, J. C. Ranasinghesagara, H. Lu, K. Mathewson, A. Walsh and R. J. Zemp, "Combined photoacoustic and ultrasound biomicroscopy," Opt. Express 17(24), 22041-22046 (2009) 